Draft VERSION July 10, 2018

Preprint typeset using $\mathrm{LT}_{\mathrm{E}} \mathrm{X}$ style emulateapj v. 5/2/11

\title{
STATISTICS OF RECONNECTION-DRIVEN TURBULENCE
}

\author{
G. Kowal ${ }^{1,2}$, D. A. Falceta-Gonçalves ${ }^{2}$, A. Lazarian ${ }^{3}$, \& E.T. Vishniac ${ }^{4}$ \\ Draft version July 10, 2018
}

\begin{abstract}
Magnetic reconnection is a process that changes magnetic field topology in highly conducting fluids. Within the standard Sweet-Parker model, this process would be too slow to explain observations (e.g. solar flares). In reality, the process must be ubiquitous as astrophysical fluids are magnetized and motions of fluid elements necessarily entail crossing of magnetic frozen in field lines and magnetic reconnection. In the presence of turbulence, the reconnection is independent of microscopic plasma properties, and may be much faster than previously thought, as proposed in Lazarian \& Vishniac (1999) and tested in Kowal et al. (2009, 2012). However, the considered turbulence in the LazarianVishniac model was imposed externally. In this work we consider reconnection-driven magnetized turbulence in realistic three-dimensional geometry initiated by stochastic noise. We demonstrate through numerical simulations that the stochastic reconnection is able to self-generate turbulence through interactions between the reconnection outflows. We analyze the statistical properties of velocity fluctuations using power spectra and anisotropy scaling in the local reference frame, which demonstrates that the reconnection produces Kolmogorov-like turbulence, compatible with Goldreich \& Sridhar (1995) model. Anisotropy statistics are, however, strongly affected by the dynamics of flows generated by reconnection process. Once the broad turbulent region is formed, the typical anisotropy scaling $l_{\|} \propto l_{\perp}^{2 / 3}$ is formed, especially for high resolution models, were the broader range of scales is available. The decay of reconnection outflows to turbulent-like fluctuations, characterized by different anisotropy scalings, strongly depends on $\beta$ plasma parameter. Moreover, the estimated reconnection rates are weakly dependent on the model resolution, suggesting that no external processes are required to make reconnection fast.
\end{abstract}

\section{INTRODUCTION}

Magnetic reconnection is a key problem for the magnetohydrodynamic (MHD) theory. It describes the interaction of magnetic flux tubes, and in particular it describes what happens when magnetic flux tubes cross each other. It is impossible to fully predict how magnetic fields evolve in turbulent environments, ubiquitous in astrophysical fluids, without a solution to this question.

The theory of magnetic reconnection can be traced back to the classical Sweet-Parker scheme (Parker 1957; Sweet 1958), for which the magnetic fluxes of opposite polarity are brought into contact over an extended region parallel to the flux and characterized by the scale $\lambda$, while experience diffusion and annihilation over the region of thickness $\delta$. This $\delta$ determines the scale of the mass outflow, that happens with the Alfvén speed $V_{A}$. Using mass conservation one can estimate the speed of crossing flux as $V_{\text {rec,SP }} \approx V_{A} \delta / \lambda$. With $\delta$ determined by microphysics and $\lambda$ being an astrophysical scale it is evident that the Sweet-Parker reconnection rate is negligibly small for most of astrophysical conditions. Indeed, taking into account that $V_{r e c, S P}$ is determined by Ohmic diffusion, i.e. $V_{\text {rec }, S P} \approx \eta / \delta$, where $\eta$ is the resistivity, one gets the

${ }^{1}$ Núcleo de Astrofísica Teórica, Universidade Cruzeiro do Sul, R. Galvão Bueno, 868 - Liberdade, CEP: 01506-000, São Paulo, $\mathrm{SP}$, Brazil

2 Escola de Artes, Ciências e Humanidades, Universidade de São Paulo, Av. Arlindo Béttio, 1000 - Ermelino Matarazzo, CEP: 03828-000, São Paulo - SP, Brazil

${ }^{3}$ Department of Astronomy, University of Wisconsin, 475 North Charter Street, Madison, WI 53706, USA

${ }^{4}$ Department of Physics \& Astronomy, Johns Hopkins University, 3400 N. Charles Street, Baltimore, MD 21218, USA classical Sweet-Parker rate $V_{\text {rec, } S P} \approx V_{A} S^{1 / 2} \ll V_{A}$ with $S=L V_{A} / \eta$ being the Lundquist number, where $L$ is a scale of the flow. Given the highly nature of astrophysical plasmas, Sweet-Parker reconnection predicts that reconnection occurs indeed very slowly.

For most of the astrophysical environments the SweetParker reconnection essentially means virtually no reconnection at finite timescales, which comes in contradiction with the observations of e.g. solar flares, which the most natural explanation is based on fast magnetic reconnection. Fast in this situation means that magnetic reconnection rate $V_{\text {rec }}$ must not depend, or at least exhibits very weak, e.g. logarithmic, dependence on the Lundquist number $S$.

Different suggestions have been made to solve the problem of fast reconnection. They included solutions that involve making $\lambda$ small, i.e. similar to $\delta$ by bending magnetic field towards the reconnection point at a sharp angle, as an ingenious suggestion by Petschek (1964). The corresponding magnetic configuration was termed "X-point reconnection". A list of other solutions where the magnetic reconnection can achieve large speeds due to a particular configuration of magnetic fluxes can be found in the book by Priest \& Forbes (2007).

The main limitation of these models is that they deal with the reconnection in rather special environments and do not address the problem of magnetic reconnection in generic astrophysical/turbulent circumstances. Could turbulence make reconnection fast? This issue was discussed in a number of papers. While Jacobson \& Moses (1984) dealt with the effects of turbulence on Ohmic resistivity, therefore decreasing $S$ by some factor, 
Matthaeus \& Lamkin (1985, 1986) performed 2D numerical simulations of MHD turbulence and claimed that the formation of X-points in 2D made the reconnection fast. Due to significant differences of MHD turbulence nature in $2 \mathrm{D}$ and $3 \mathrm{D}$, as well as the ambiguity of reconnection rate measurements within the $2 \mathrm{D}$ distribution of turbulent magnetic flux, these interesting approaches could not be directly compared to real astrophysical scenarios.

A following model that related the modern theory of 3D MHD turbulence, i.e. the Goldreich \& Sridhar (1995) one, and magnetic reconnection was proposed in Lazarian \& Vishniac (1999, henceforth LV99). There the outflow region $\delta$ is not determined by microscopic diffusive processes, but by the wandering of magnetic field lines. The prediction of the LV99 theory was that the reconnection changes with the level of turbulence. This quantitative prediction was successfully tested in the numerical studies Kowal et al. (2009, 2012) investigating the effects of resolution, explicit resistivity, ways of turbulence is driven, and strengths of guide field. More recently, another confirmation of LV99 came from relativistic MHD simulations by Takamoto et al. (2015). Observational testing of the theory are discussed e.g. in recent reviews by Lazarian et al. (2015, 2016).

The LV99 predicts that reconnection is fast, i.e. does not depend on $S$, in generic astrophysical conditions. It predicts that reconnection happens not only at particular places where the magnetic field lines happen to undergo a special configuration, but through the entire turbulent volume. In fact, the theory makes the MHD turbulence theory by Goldreich \& Sridhar (1995) selfconsistent and predicts the violation of the classical magnetic flux freezing Alfvén (1942) ${ }^{5}$. The violation of flux freezing in turbulent fluids entails many vital astrophysical consequences (see Lazarian 2005; Santos-Lima et al. 2010 Lazarian et al. 2012) and it has been explored theoretically Eyink (2011); Eyink et al. (2011) and confirmed numerically Eyink et al. (2013).

While turbulence is really ubiquitous in astrophysical environments (see Armstrong et al. 1995, Padoan et al. 2009. Chepurnov \& Lazarian 2010 Chepurnov et al. 2015), a question arises of whether reconnection itself could induce turbulence that would make the it fast. This possibility was mentioned in LV99 and quantified in Lazarian \& Vishniac (2009) within the model of flares of magnetic reconnection in high $\beta$-plasma environment ${ }^{6}$. The first numerical simulations of magnetic reconnection induced by turbulence that is generated by reconnection were performed in Beresnyak (2013) under incompressible approximation, and in Oishi et al. (2015) and Huang \& Bhattacharjee (2016) using compressible codes. However, these works did not consider a few studies which we address here. For instance, Beresnyak (2013) \& Oishi et al. (2015) did not perform the studies of turbulence properties and anisotropy statistics. Although Huang \& Bhattacharjee (2016) included these studies, their setup was significantly different, and the statistics were calculated at relatively earlier time $t=3.5 t_{A}$. Moreover, none of these works addressed the question of turbulence de-

\footnotetext{
${ }^{5}$ The violation of flux freezing is implicit in LV99 but is explicitly treated in the subsequent proceedings paper Vishniac \& Lazarian (1999).

${ }^{0}$ Plasma $\beta$ is the ratio of the thermal to magnetic pressure.
}

velopment under different $\beta$-plasma parameters.

In this paper we aim to understand the reconnectiondriven turbulence in compressible MHD framework, focusing on long time evolution and turbulence statistics changes during the whole simulation. For that we present a number of high resolution numerical experiments, for which we analyze and interpret the properties of obtained turbulent velocity fluctuations. We demonstrate the dependencies on the resolution and $\beta$-plasma. In Section 2 we present our methodology and preformed numerical models. In Section 3 we describe obtained results from the analysis of temporal evolution and statistical properties of generated turbulent fluctuations. In Section 4 we discuss our results and compare them to the previously done models. Finally, in Section 5 we draw our conclusions.

\section{METHODOLOGY AND MODELING}

We use a high-order shock-capturing Godunov-type code AMUN7 based on the adaptive mesh. The code integrates the set of isothermal compressible magnetohydrodynamic (MHD) equations

$$
\begin{array}{r}
\frac{\partial \rho}{\partial t}+\nabla \cdot(\rho \boldsymbol{v})=0 \\
\frac{\partial \rho \boldsymbol{v}}{\partial t}+\nabla \cdot\left[\rho \boldsymbol{v} \boldsymbol{v}+\left(a^{2} \rho+\frac{B^{2}}{8 \pi}\right) I-\frac{1}{4 \pi} \boldsymbol{B} \boldsymbol{B}\right]= \\
\nu \nabla^{2}(\rho \vec{v}) \\
\frac{\partial \boldsymbol{B}}{\partial t}+\nabla \times \boldsymbol{E}=0
\end{array}
$$

where $\rho$ and $\boldsymbol{v}$ are plasma density and velocity, respectively, $\boldsymbol{B}$ is the magnetic field, $\boldsymbol{E}=-\boldsymbol{v} \times \boldsymbol{B}+\eta \boldsymbol{J}$ is the electric field, $\boldsymbol{J}=\nabla \times \boldsymbol{B}$ is the current density, $a$ is the isothermal speed of sound, and $\nu$ and $\eta$ are the viscosity and resistivity coefficients, respectively.

We integrated the governing equations using the $3^{\text {rd }}$ order 4-stage Strong Stability Preserving Runge-Kutta (SSPRK) method (Ruuth 2006), a 3D spatial Gaussian processes based reconstruction limited near extrema (Kowal 2016), and a multi-state Harten-Lax-van Leer (HLLD) approximate Riemann solver Mignone (2007). In order to keep $\nabla \cdot \vec{B}$ negligible, we solve the induction equation (Eq. 3) using the hyperbolic divergence cleaning based on generalized Lagrange multiplier (GLM) method (Dedner et al. 2002). This set of numerical algorithms, together with the stability CFL-coefficient set to 0.3 for all models, results in stable numerically modeled data of high quality.

We performed a number of numerical simulations within a 3D domain with physical dimensions $1.0 \times$ $4.0 \times 0.5$ with its center placed at Cartesian coordinates $(0,0,0)$. Our code make use of adaptive mesh, for which the refinement criterion is based on the magnitude of the local vorticity $|\vec{\omega}|=|\nabla \times \vec{V}|$ and current density $|\vec{J}|$. The thresholds determining the refinement and derefinement of the mesh are set to 0.1 and 0.01 , respectively, indicating that the mesh is quickly refined in the turbulent and reconnection regions. The base domain is divided into $2 \times 8 \times 1$ blocks, each with $32^{3}$ resolution, from which we refine the mesh using the above mentioned criterion

7 The code is freely available at http://amuncode.org 


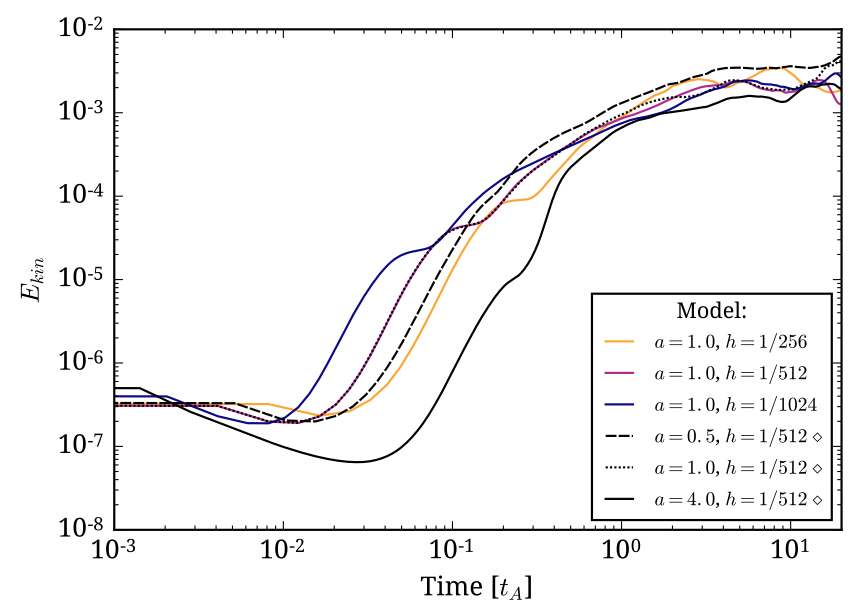

FIG. 1. - Evolution of kinetic energy density for models with different resolutions and sound speeds. Depending on the grid size $h$ or $\beta$ plasma parameter (determined by sound speed $a$ ) the growth of kinetic energy starts at different times. In all cases, the kinetic energy grows from the initial value of around $5 \cdot 10^{-7}$ by more than three orders of magnitude, independently of $h$ or $\beta$. Models with different sound speeds $a$ are indicated by symbol $\diamond$. They are also performed in a box with $L_{x}=L_{z}=1.0$.

up to 3,4 , and 5 refinement levels with the resulting effective grid sizes $\Delta x=\Delta y=\Delta z=h=1 / 256,1 / 512$, and $1 / 1024$, respectively. For the later, the effective resolution is $1024 \times 4096 \times 512$, therefore. For models with different $\beta$-plasma parameters, indicated with $\diamond$ in the figures, we use box which is squared in the XZ-plane, i.e. its physical dimensions are $1.0 \times 4.0 \times 1.0$, with the maximum refinement level equal to 4 , and the effective resolution $512 \times 2048 \times 512$.

The initial magnetic field configuration is antiparallel along the $\mathrm{X}$ direction with the magnitude equal to 1.0 and a discontinuity placed at the XZ plane $y=0$. Additional guide field is imposed along the $\mathrm{Z}$ direction with a uniform amplitude 0.1. Uniform initial density is set to 1.0 in the whole computational domain. The initial velocity perturbation with random distribution of directions and maximum amplitude of $0.0173^{8}$ is set in the region within the distance of 0.1 from the initial magnetic field discontinuity. We set the sound speed $a$ to $0.5,1.0$ or 4.0 , which give plasma parameter $\beta=p_{\text {th }} / p_{\text {mag }} \approx 0.5$, 2.0 or 32.0 , respectively.

We do not set viscosity and resistivity explicitly. The dissipation of kinetic and magnetic energies happens through the numerical diffusion. From earlier models (see Kowal et al. 2009, 2012) we estimated the numerical resistivity to be smaller than $3 \cdot 10^{-4}$ in models with the grid size $h=1 / 256$, resulting in Lundquist number $S \approx 3 \cdot 10^{3}$ or higher for models with the grid sizes presented here. Since the same scheme was applied in the solution of all MHD equations, we can assume that the numerical viscosity and resistivity are similar, which gives the Prandtl number $P r_{m}=\nu / \eta$ of the order of one.

We applied periodic boundary conditions along the $\mathrm{X}$ and $\mathrm{Z}$ directions and open (normal derivatives to the boundary plane are set to zero) ones along the $\mathrm{Y}$ direction. This choice of boundary conditions corresponds

8 Each component of velocity has a uniform random distribution between -0.01 and 0.01 resulting in a non-uniform velocity magnitude distribution with a maximum value of $0.01 \sqrt{3}$.

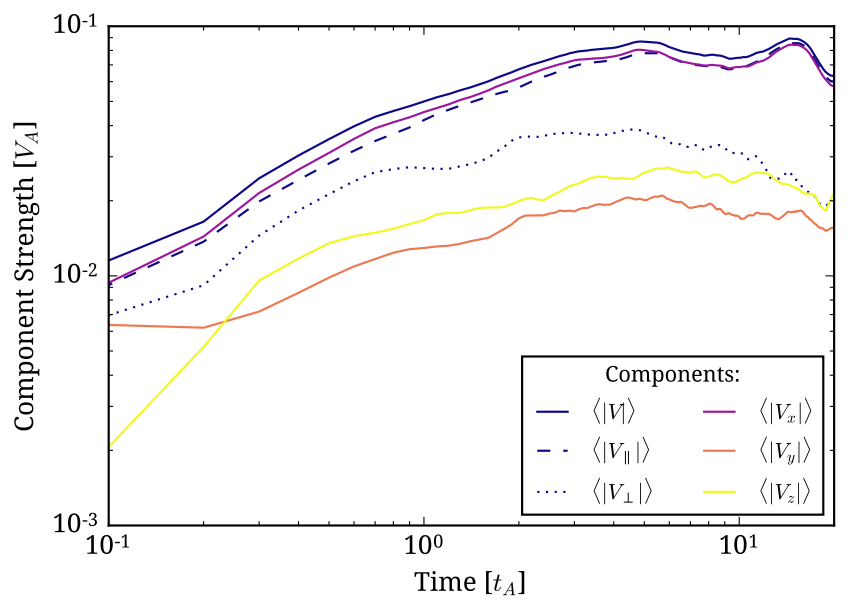

FIG. 2.- Evolution of standard deviation of the velocity and its components: X, Y, Z, and parallel and perpendicular to the local magnetic field for model with sound speed $a=1.0$ and the grid size $h=1 / 512$.

to a subvolume of a larger box with a size $L \gg 1.0$ and imposes certain restrictions on applications, which we discuss more extensively in the discussion section.

\section{ANALYSIS AND RESULTS}

\subsection{Turbulence Evolution}

We started our models with a weak random velocity fluctuations of the order of $1 \%$ of the Alfvén velocity, which launches the magnetic reconnection process within the initial current sheet. This process normally converts magnetic energy into kinetic one and heat. Since we used the isothermal equation of state, the set up corresponds to a situation that the heat is quickly dissipated.

The first question we want to answer is, if the process of magnetic reconnection can sustain the injection of kinetic energy to the system or if the initial velocity fluctuations simply decay due to the dissipation. In order to answer it, we analyze our result first in terms of global quantities, such as the kinetic energy evolution, the evolution of velocity component contribution to the kinetic energy, the evolution of total vorticity generated by the reconnection, and the change of the volume fraction characterized by a high vorticity value.

Figure 1 shows the evolution of kinetic energy density integrated over the whole domain, up to the final simulation time $t=20.0$. The initial value of the energy for all models is about $5 \cdot 10^{-7}$. We shall remind, that the turbulence is generated only in the vicinity of current sheet by the action of magnetic reconnection. Therefore, it can be dynamically important there. In most of the domain volume, however, the kinetic energy remains negligible. The figure demonstrates a quick growth of $E_{k i n}$ to values above $10^{-3}$ at relatively short time of one Alfvén time unit. This indicates an increase of the kinetic energy by almost three orders of magnitude. We should note, that the initial velocity perturbations were generated from a random noise, therefore they are strongly dissipated by the numerical dissipation during an initial period of about $0.02 t_{A}$, causing a small drop of kinetic energy, and reducing the strength of fluctuations interacting with the current density, as seen in Figure 1. This drop depends on the used grid size $h$ and sound speed $a$. For example, it is bigger and reaches the minimum at slightly later 
times (the minimum appears around $0.03 t_{A}$ ) for model with the sound speed equal 4.0 (black line), comparing to model with $a=1.0$. Even though these fluctuations are weakened, they are still strong enough to sufficiently deform the current sheet locally, which results in development of instabilities. For the scope of this manuscript, we do not identify the type of instability, and just focus on the characteristics turbulence produced as a result. After around $1 t_{A}$, the levels of kinetic energy are comparable for models with different sound speeds $a$ (or $\beta$ ) and grid sizes $h$.

How this energy is distributed among different velocity components? In Figure 2 we show the standard deviation of the total velocity, its $\mathrm{X}, \mathrm{Y}$, and $\mathrm{Z}$ components, and components parallel and perpendicular to the local field for model with sound speed $a=1.0$ and the grid size $h=1 / 512$. Initially, the X, Y, and Z-components of velocity are equal, but shortly the $\mathrm{X}$ component, which is in the direction of the antiparallel magnetic field $B_{x}$, starts to dominate. Comparing to the kinetic energy evolution, we see that this component caries most of the energy. The Z-component is slightly stronger than the $\mathrm{Y}$ one, since we imposed non-zero guide field in the system. Comparing components parallel (dashed) and perpendicular (dotted) to the local field, we see that the parallel one is developing to values a few times larger than the perpendicular one. This indicates that the strongest motion generation takes place along the mean magnetic field and is associated with the reconnection process. A similar behavior is observed for two components, $V_{x}$ and $V_{\|}$.

The evolution of kinetic energy and velocity components described above clearly demonstrates that the reconnection is able to generate significant plasma motions, which could dominate the local dynamics. Below, we investigate more closely what type of motions we observe in our models, turbulent or laminar. The simplest way of detecting turbulent eddies in a system is by calculating the vorticity, $\vec{\omega}=\nabla \times \vec{v}$, which directly measures the rotational motions. In the first analysis, we calculate the magnitude of vorticity and average it over the computation domain at each time step. We also analyze the reconnection rate estimated using two different methods. Later, we analyze their spectral properties and anisotropy.

The top left plot of Figure 3 shows the average of vorticity magnitude $|\vec{\omega}|$ as a function of time for all models. The amount of turbulent motions generated due to reconnection is growing until it reaches a saturation at around $t=5.0$. The vorticity growth rate seems to gradually decrease with time until the saturation. Since the reconnection works at smallest scales, the vorticity growth depends on the resolution, what can be seen by different maximum values of averaged vorticity, $|\vec{\omega}| \approx 4.0,5.0$, and 8.0 for models with grid sizes $h=1 / 256,1 / 512$, and $1 / 1024$, respectively. This dependence with the grid size is not linear and may indicate that for smaller grid sizes even higher vorticities can be generated. After reaching the maximum, the vorticity generation slowly decays for models with medium and large grid sizes. It increases for the model with the highest resolution, however. In three models with different sound speeds (dashed, dotted, and solid black lines corresponding to $a=0.5,1.0$, and 4.0, respectively), the total vorticity grows through the whole simulation, but its value is $a$ dependent, i.e. it decays with $a$ (or $\beta$ ).

The top right plot of the same figure shows the filling factor for vorticity $|\vec{\omega}| \geq 10.0$. It simply shows a volume where vorticity is equal or larger than the threshold, and indicates how the volume of strong rotational motions changes. The chosen threshold can be interpreted as a change of velocity by $0.1 V_{A}$ within a distance 0.01 , which corresponds to relatively strong rotation or shear. In our simulations, we observe peak values of vorticity as high as a few hundreds, related to very strong reconnection events (change of the order of $V_{A}$ at the grid size scale). The top right plot in Figure 3 demonstrates the initial nearly linear growth of the volume occupied by relatively strong rotational motions, almost independent of the used resolution or sound speed, until about $t=6.0$. This moment, at which the growth rate of the filling factor suddenly changes, is also independent on the effective resolution $h$. The maximum value, however, is resolution sensitive, with the highest volume observed in the model with the smallest grid size. The evolution of filling factor after this moment have different behavior for different resolution, as well. For the model with the largest grid size, the filling factor starts to decay after $t \approx 8$, for middle grid size it stay nearly constant until $t \approx 16$, and for the smaller grid size, it seems to continue growing. This indicates that in order to resolve well the turbulence we have to use the highest resolution possible. The filling factor evolution seems to be very weakly dependent on plasma $\beta$ parameter. Comparing models with different $a$ and the same grid size $h=1 / 512$, we see that the filling factor follows similar evolution growing in all cases, but reaching different filling factor values for different sound speeds. Comparing to the model with $a=0.5$ (dashed line) to models with $a=1.0$ and $a=4.0$, the filling factor reaches values by $5-20 \%$ and $20-30 \%$ lower, respectively, after $t=5.0$.

In two bottom plots of Figure 3 we show two ways of estimating the reconnection rate. In the right plot of Figure 3 we present the evolution of the estimated thickness of the turbulent layer $\Delta$. The thickness was calculated from the region where the magnitude of vorticity is above a threshold, in our case $|\vec{\omega}| \geq 10.0$. As we see, initially the thickness continues to grow linearly, almost independently of the grid size, and after $t \approx 6.0$ its growth slows down. For the lowest resolution model $(h=1 / 256)$, it continues to grow up to $t=9.0$ and then saturates. In the case of medium resolution model $(h=1 / 512)$, $\Delta$ continues to increase until $t \approx 16$, and for the highest resolution model $(h=1 / 1024)$ it increases with the same rate until the end of simulation. For models with different sound speeds, the turbulent region thickness grows during the whole simulation. In the subplot of this plot, we show reconnection rates for all models estimated from the growth rate of the turbulent region thickness $V_{\text {rec }}=d \Delta / d t$ within the initial period $t \leq 5.0$, in the same way as it was done by Beresnyak (2013). The $\times$ points correspond to models with different $\beta$, while the + points are for models with different resolutions and the same $a=1.0$. We see that the reconnection rate depends slightly on $\beta$ plasma, decreasing with its value. We estimated $V_{\text {rec }}$ to be about $0.0327 \pm 0.0003$, $0.0302 \pm 0.0002$, and $0.0255 \pm 0.0003$ for $\beta \approx 0.5,2.0$, and 32.0 , respectively. From these points we estimated an empritical dependence $V_{\text {rec }}(\beta)=-0.0017 \log \beta+0.0314$, 

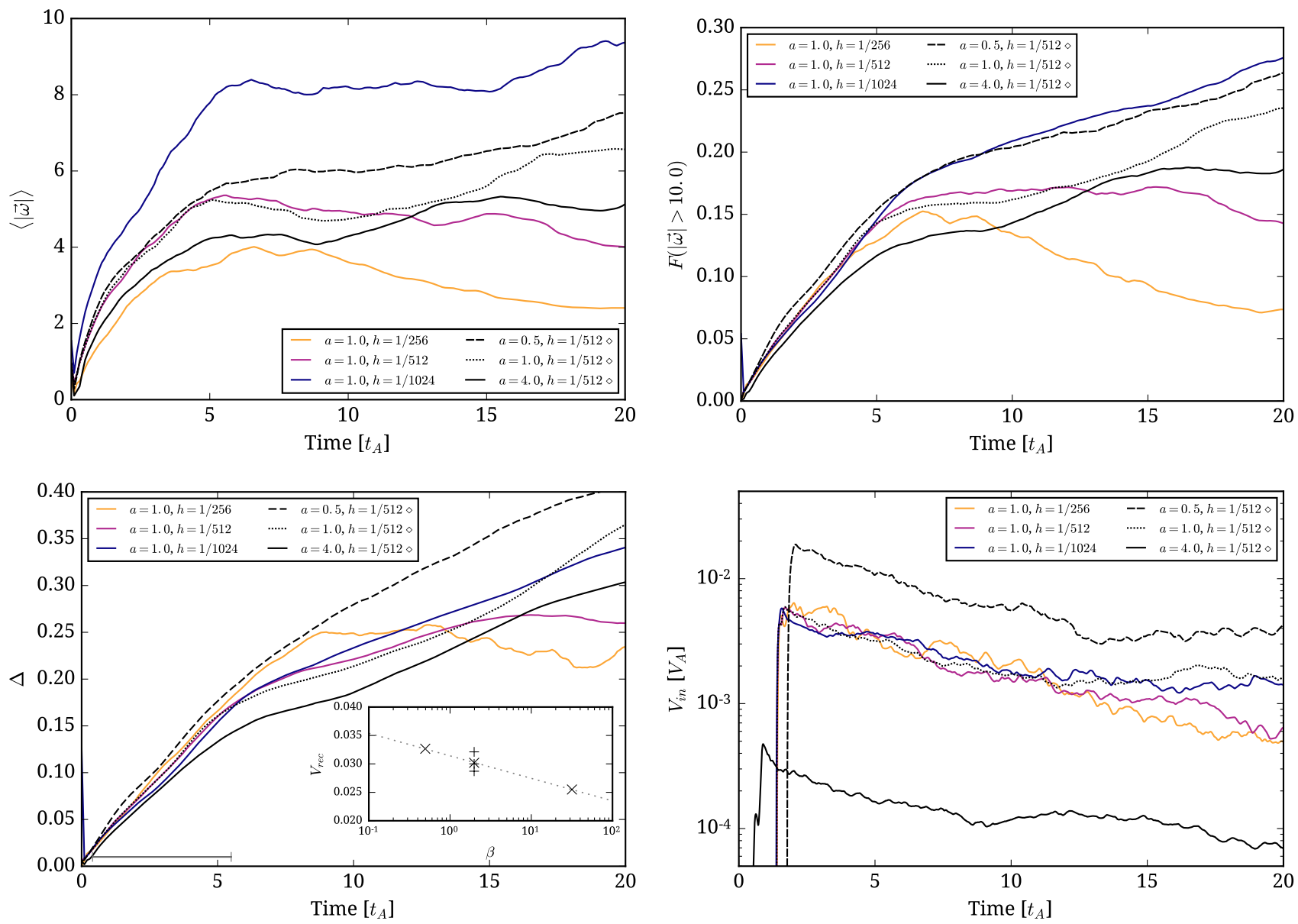

FIG. 3. - Evolution of the mean vorticity for models with different resolutions (top left) and the filling factor of volume where the vorticity is equal or larger than a given threshold (top right). The plot shows the absolute value of vorticity averaged over the simulation box for models with different resolutions. In the bottom left plot we show the evolution of the turbulent region thickness $\Delta$ for models with different resolutions. In the subplot of this plot we estimated the reconnection rate measured by the growth rate of the turbulent region thickness $V_{r e c}=d \Delta / d t$, in a similar way as Beresnyak (2013). The estimation was done within the period indicated by the horizontal gray line. In the bottom right plot we show the average inflow speed $V_{i n}$, i.e. the speed with which the new magnetic flux is brought into the box through the top and bottom open boundaries. This speed is expressed in $V_{A}$ and is useful to estimate the magnetic field dissipation rate. As we can see, $V_{i n}$ seems to be independent of the grid size (or numerical dissipation), but is strongly dependent on sound speed $a$.

with both coefficients rounded to the fitting error. This weak dependence could indicate the importance of supersonic motions on bending magnetic field lines within the current sheet. There the reconnection outflows can approach the Alfvén speed, which means that for the low $\beta$ plasma, the outflows become supersonic. Also, the magnetic field strength is reduced, therefore its easier to bend and effectively reconnect its lines.

The reconnection rate $V_{\text {rec }}$ also weakly depends on the used resolution, i.e. it grows with the grid size $h$. The resolution dependence could be related to the numerical resistivity, which is proportional to $h$, causing the reconnection efficiency to be higher for models with bigger $h$. For the model with the highest resolution $(h=1 / 1024)$, the estimated reconnection rate is about $0.0287 \pm 0.0003$, nearly twice as much as the value estimated by Beresnyak (2013). Remembering that Beresnyak (2013) used much less dissipative spectral code and smaller values of the resistivity, our result should be compatible with theirs.

As we indicated, the reconnection rates presented in the bottom left plot were estimated for times $t \leq 5.0$. At later times, the growth rate of turbulent regime depends on the grid size $h$. Once the turbulence is fully developed, we should expect the reconnection to perform more efficiently, as predicted by LV99. The observed tendency is that for $t \geq 5.0$ the reconnection rate estimated using the thickness of turbulent region decreases with $h$, and even statures in the case of the lowest resolution model $(h=1 / 256)$. For the highest resolution model, the turbulent region thickness $\Delta$ increases all the time, yet its growth rate is not as high as at earlier period $t \leq 5.0$. In real systems, however, where the resolution is virtually infinite, we should expect that the thickness of turbulent region grows constantly with the same rate for later times too. In models with different sound speeds we also see decrease of the growth rate of the turbulent region thickness after $t \approx 5.0$, however, it is roughly independent of the used $\beta$.

In the bottom right plot of Figure 3 , we show the mean inflow speed $V_{\text {in }}$ measured at the upper and lower boundaries at $y= \pm 2.0$. Since these boundaries are open, the magnetic flux can be freely transported through them. The positive values of $V_{i n}$ indicate that the velocity at the boundary is directed toward the interior of the domain, therefore, it brings fresh magnetic flux to the system. In the standard Sweet-Parker model we interpret 

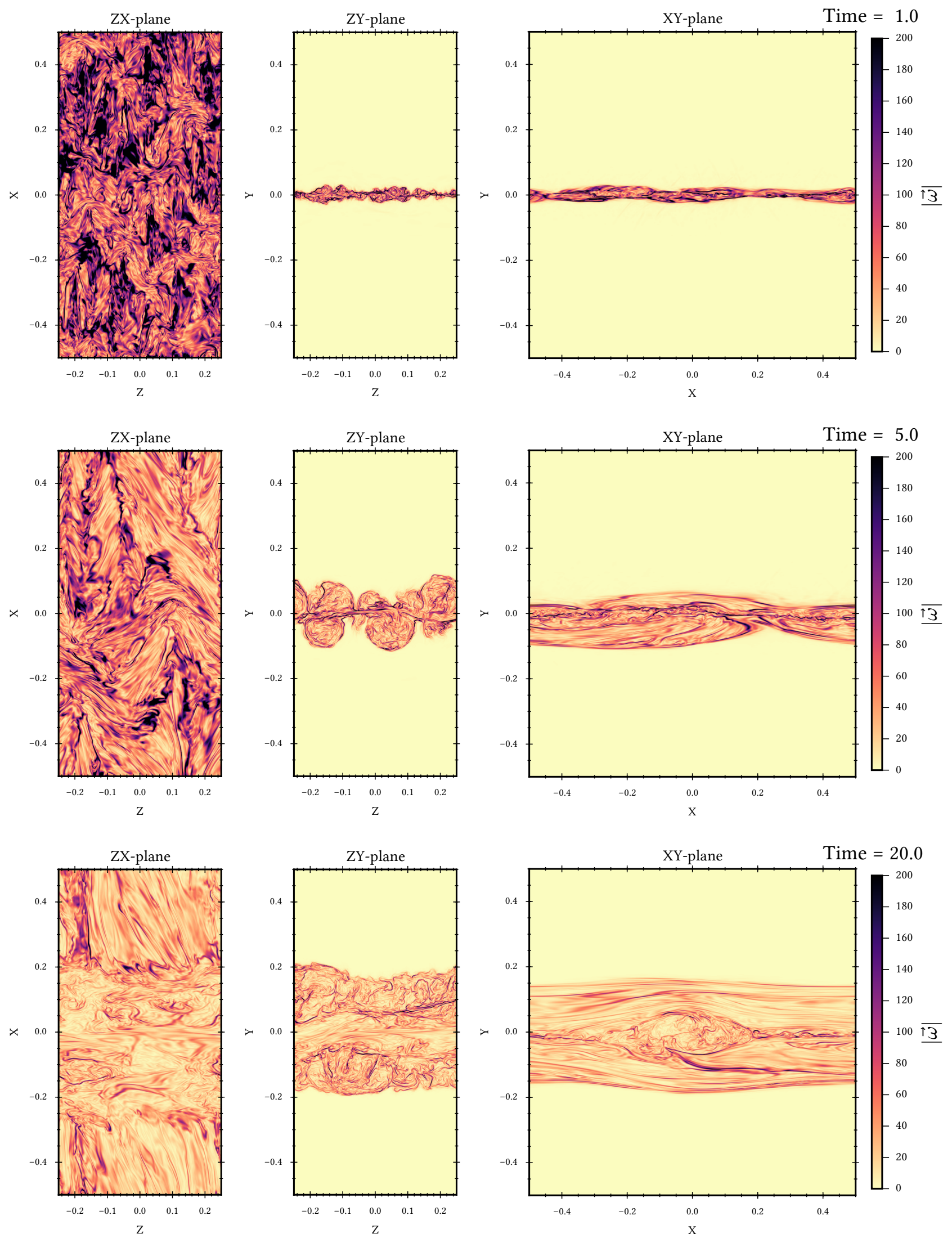

FIG. 4. - Vorticity amplitude slices along three main planes, ZX (left), ZY (center), and XY (right) for three different moments in time, $t=1.0,5.0$, and 20.0 (top, middle, and bottom, respectively) for model with sound speed $a=1.0$ and grid size $h=1 / 1024$. The turbulent region develops near the initial current sheet and expands in the Y direction. The plots show subregions limited to $y \leq 0.5$ from the whole domain extended up to $y=2.0$. 

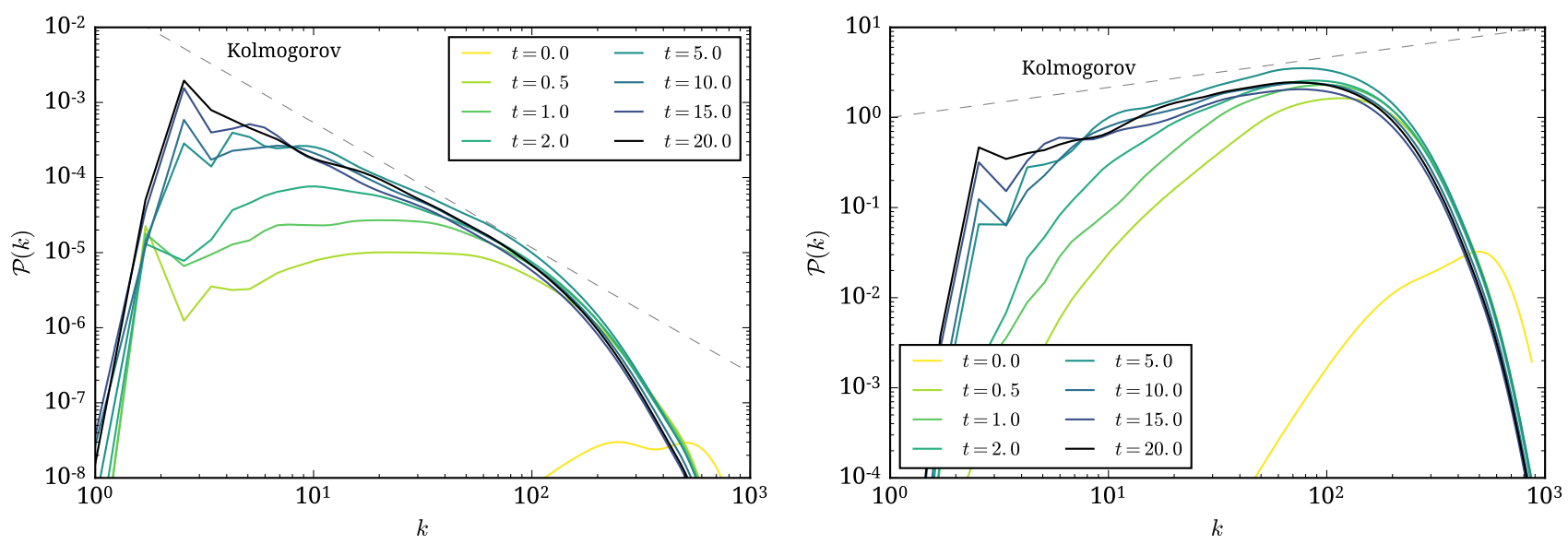

FIG. 5.- Evolution of power spectra of velocity (left) and vorticity (right) for model with the grid size $h=1 / 1024$. We plot several power spectra calculated at different times.

this quantity as a measure of the reconnection process efficiency. The only way magnetic field can be changed such system is by its conversion into other forms of energy, dissipation, or transport through the open boundaries. Since our inflow boundaries are far from the initial current sheet, we observe a zero inflow during the initial period, followed by a very quick burst of the inflow and then a decay. The maximum value of $V_{i n}$ and its following decay seems to be nearly independent of the grid size $h$. It indicates that the reconnection process under the same physical conditions is independent of numerical dissipation, even if its rate reaches values which are only small factors of $V_{A}$. These small values of $V_{i n}$ are mostly due to the fact, that our boundaries along the $\mathrm{X}$ direction are periodic, so the reconnected flux is not removed from the system. Comparing models with different sound speeds $a$ (or plasma $\beta$ parameters) we see that the inflow speed $V_{i n}$ is very sensitive to $a$. The moment at which $V_{i n}$ starts to quickly grow happens at different times for models with different $a$, and $V_{i n}$ reaches significantly different values at later times. We verified that within the turbulent region, the maximum sonic Mach number reaches around 2.0 for model with $=0.5$, and around 1.0 or below 0.25 for models with $a=1.0$ and 4.0 , respectively, signifying that the supersonic turbulence can be generated in models with $a<1.0$. This means that the compression may be "squeezing" more magnetic field within the turbulent region and/or it is more efficiently dissipated in local shocks. This process would saturate in the presence of open boundary conditions along the current sheet allowing for removal of the reconnected magnetic field. However, with the periodic boundaries, the flux is accumulated and therefore the inflow speed may not be a reliable measure of the reconnection rate in these systems.

Figure 4 shows slices of total vorticity along the three main mid-planes at three different moments, $t=1.0,5.0$, and 20.0. All plots have the same color range for easier comparison and are obtained from the model with the smallest grid size $h=1 / 1024$. We see that the stochastic reconnection develops complex filamentary structures near the current sheet, increasing their volume with time, what supports the filling factor evolution shown in the right plot of Figure 3 It is important to recognize, that most of the turbulent motions are developed in the ZY- plane perpendicular to the reconnecting field. Vorticity shows places in which the velocity changes quickly. As we see in the middle column (the ZY-plane), the high vorticity filaments have arbitrary orientations. In the right plots (the XY-plane), however, the filaments tent to align with the $\mathrm{X}$ direction, especially at later times (central and bottom plots). This is explained well by the fact that the motions can mix reconnecting field lines easier in the perpendicular plane than in the parallel direction, due to the magnetic tension, even if these motions are produced by the reconnection of the same lines. These visualizations demonstrate how a simple current sheet with a weak velocity noise can create complex turbulent structure within a broad vicinity of it.

\subsection{Turbulence Properties: Power Spectra and Anisotropy}

In the previous subsection we studied the evolution of turbulent motions produced by stochastic reconnection and initiated from a random velocity noise. We analyzed how the kinetic energy and vorticity evolves with time, what is the growth rate of the turbulent region thickness and how the estimated reconnection rate depends on the used grid size or $\beta$. Here we take a closer look into the statistical properties of turbulent motions by studying their power spectra and anisotropy. The fundamental question we want to answer here is if the turbulence generated by reconnection can be characterized a by a mixture of strong turbulence, described by Goldreich \& Sridhar (1995) model, and structures produced by the reconnection ejection regions.

In Figure 5 we show power spectra of velocity (left) and vorticity (right) for different moments during the turbulence development. The power spectra were calculated using 3D wavelets in Fourier space (see Kirby 2005). For comparison, we show Kolmogorov slope $\left(k^{-5 / 3}\right.$ for velocity, and corresponding $k^{1 / 3}$ for vorticity) using gray dashed line. The initial power spectrum $(t=0.0)$ is shown using the brightest line (yellow in the color version). The velocity fluctuations are spread over the smallest scales with very small amplitudes $\left(P(k)<10^{-7}\right.$ for velocity, see the left plot). Fluctuations of both quantities, velocity and vorticity, quickly develop from small to large scales. At $t=0.5$, they are already spread over 

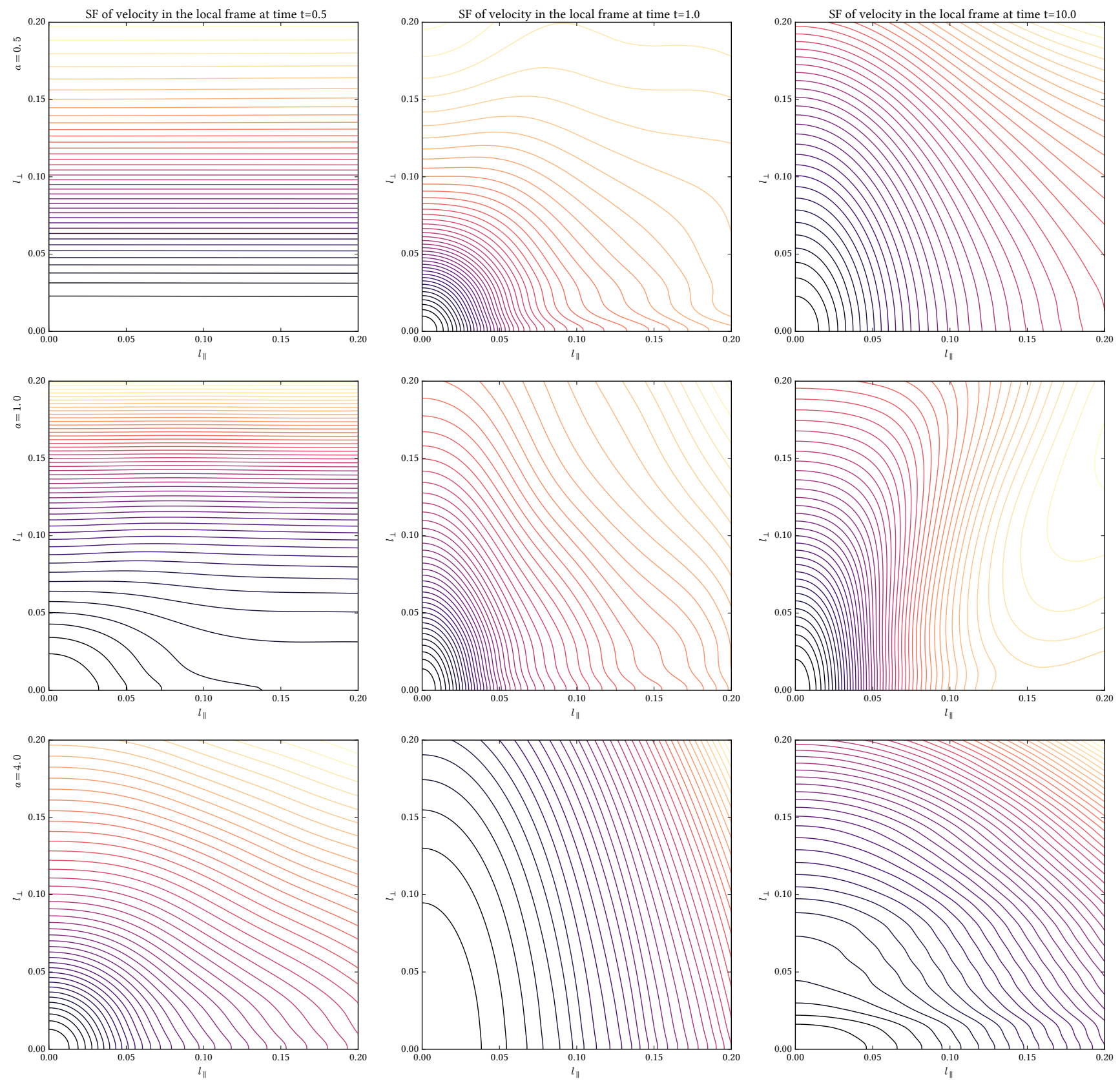

FIG. 6.- Anisotropy of velocity structure functions with respect to the local mean magnetic field at two early time moments $t=0.5$ (left) and $t=1.0$ (center), and later one at $t=10.0$ (right). Structure functions were calculated for the model with the grid size $h=1 / 512$ and box size $L_{x}=L_{z}=1.0$. The upper, middle and lower rows correspond to models with sound speed $a=0.5,1.0$ and 4.0 , respectively.

large range of scales (up to $k \leq 10$ ). After $t>2.0$, power spectra start to align with the Kolmogorov slope forming the inertial range. At $t=5.0$ the inertial range is well developed and it extends from $k \approx 10$ down to nearly $k=200$ for models with the effective grid size $h=1 / 1024$. At the final moment of simulation, $t=20.0$, the power spectrum is fully developed and stationary (compare its change for $t \geq 10$ ), both for velocity and vorticity, and is characterized by a slope close to the Kolmogorov one.

The development of broad power spectrum from small to large scales cannot be simply explained by reconnection which usually operates at small scales across the current sheet. If considered one reconnection event, the ejection occurs along a thin slab determined by the local current sheet thickness. However, within the local ejection region, the magnetic field is oriented perpendicularly to the current sheet plane, since the reconnected component is removed along the slab. On the other hand, a typical fluctuation scale along the current sheet is determined by the local separation of the reconnection events. Initially, this separation scale is very short in our case due to densely packed reconnection events, and later it increases with time due to the interactions between ejections acting at larger and larger scales. This picture is well justified by plots in the left column of Figure 4, where a typical scale of vorticity structures seem to increase with time (compare ZX-plane 
cuts for different time moments). This also indicates a development of velocity anisotropy dominated by initially small and nearly isotropic structures and transformed into anisotropic ones. This picture can be confirmed if we estimate the anisotropy with respect to the mean direction of reconnecting components, i.e. along the $\mathrm{X}$ direction. However, what is the anisotropy with respect to the local mean field and the reconnecting one? Should we expect two different scalings, i.e. Goldreich \& Sridhar-like at large scales and a different one produced by reconnection outflow regions at smaller scales? Up to which scales the outflows can affect the statistics? How the anisotropy is developed for different $\beta$-plasma parameters?

In Figure 6 we show the second-order structure functions of velocity calculated in the local reference frame, i.e. with respect to the local mean magnetic field at three different time moments: $t=0.5,1.0$, and 10.0 (left, center, and right columns, respectively) for models with $a=0.5,1.0$, and 4.0 (upper, middle and lower rows, respectively). We notice the change of anisotropy degree $l_{\|} / l_{\perp}$ between different moments. For all three models with different $a$ at time $t=0.5$ the velocity fluctuations are elongated with the local magnetic field. We clearly see that the anisotropy decreases with increasing $a$ (or $\beta$ ). However, once the reconnection increases its efficiency, the velocity fluctuations start to be elongated rather in the direction perpendicular to the local field (see the central column). At $t=1.0$, the velocity fluctuations are relatively isotropic for model with $a=0.5$ (for $l<0.1$ ), while for other models they are elongated in the perpendicular direction to the local field.

Why this sudden change of orientation of anisotropic velocity structures? Our understanding is that the Alfvén waves dominate initially the structure of velocity, but the local reconnection events become quickly stronger taking over the statistics. A local reconnection event can be characterized by a weak and broad inflow of the magnetic flux, and strong and thin outflow region (see the typical Sweet-Parker picture). Within the outflow region, the velocity is oriented in the direction perpendicular to the local field (i.e. with respect to the reconnected flux component and the guide field), therefore the flow is perpendicular to this field. This is clearly observed in the right top plot of Figure 6 showing the local structure function at $t=10.0$, where its value increases quickly along the parallel direction, determining the averaged thickness of the current sheet $(\delta<0.05)$, while in the perpendicular direction it increases slowly up to a fraction of the length unit. This anisotropy continues to dominate in perpendicular direction during the later times of our simulation (see the right plots of Figure 6 for $a=0.5$ and 1.0). At later time $t=10.0$ for the model with the highest sound speed $(a=4.0)$ the anisotropy degree indicates that fluctuations change their orientation and are aligned with the local magnetic field rather, on the contrary to models $a \leq 1.0$. This indicates that the compressibility could be an important factor in determining the small scale anisotropy in reconnection-driven turbulence, since it increases with decreasing sound speed.

As we discussed above, the reconnection generate strong anisotropy in the perpendicular direction to the local field, and during the evolution the anisotropy degree changes significantly. The dominance of the perpen- dicularly oriented fluctuations is observed in the whole evolution of models with $a \leq 1.0$ and during the initial few Alfvén time units in the model with $a=4.0$. The difference comes from the fact, that the reconnection outflow interactions decay to turbulent fluctuations, and the timescale for this process seem to depend on fast magnetosonic speed, since it is faster for higher sound speeds. Of course, this results also in the same timescale for anisotropy changes. The perpendicular anisotropy dominance is in contrast to the Goldreich-Sridhar turbulence where velocity eddies are elongated with the magnetic field. We explained, that the difference comes from the strong reconnection outflows dominating the structure function, especially at small scales. However, even though the fluctuations are not oriented along the local field, maybe at least the scaling of anisotropy reveals some compatibility with the Goldreich-Sridhar model?

In Figure 7 we show anisotropy scaling of velocity fluctuations at several moments, $t=0.5,2.0,5.0,10.0$, and 20.0 for the model with $a=1.0(h=1 / 1024$, left plot $)$, and $t=0.5,1.0,2.0,5.0$, and 10.0 for the model with $a=4.0$ ( $h=1 / 512$, right plot). These plots confirm that the anisotropy degree varies significantly during the system evolution, especially at the beginning of the simulation. Initially isotropic fluctuations develop strong anisotropy at all scales along the perpendicular direction $l_{\perp}$ (shown in the horizontal axis) with scaling close to $l_{\|} \propto l_{\perp}$, but after about $t=5.0$ for the model with $a=1.0$ and $t=2.0$ for the model with $a=4.0$ the anisotropy degree tends to decrease. Even though the anisotropy changes significantly, its scaling seems to be relatively consistent up to $t=10.0$ for the model with $a=1.0$. We observe that for scales below 0.1 it continues to follow $l_{\|} \propto l_{\perp}$, while only at larger scales it starts to manifest the Goldreich-Sridhar scaling $l_{\|} \propto l_{\perp}^{2 / 3}$. On the right, the anisotropy scaling becomes compatible with $l_{\|} \propto l_{\perp}^{2 / 3}$ earlier. We see it is formed already at $t=5.0$ from $l_{\perp} \approx 0.1$ down to scales as small as 0.01. At this time, the turbulent region is relatively thick, as estimated in the bottom left plot in Figure 3 . The developed turbulence have enough space to create inertial range and structure compatible with strong turbulence. At the same time reconnection events spread sparsely over the same turbulent region, affecting only the local velocity structures and relaxing to turbulent motions at shorter scales for the model with higher sound speed. This indicates the presence of strong turbulence described by the Goldreich-Sridhar theory, substantially disturbed by the injection from the reconnection outflows at smaller scales, characterized by different properties.

\subsection{Decaying Turbulence without Global Field Reversal}

In order to remove the strong contribution of the reconnection outflows observed in the anisotropy scaling of velocity fluctuations, which is responsible for deviation from Goldreich \& Sridhar (1995) scaling in the model with sound speed $a=1.0$, we performed a run with the grid size $h=1 / 512$ in which we removed the global reversal of magnetic field by setting $\left|B_{x}\right|$ everywhere and leaving other two components, $B_{y}$ and $B_{z}$, unchanged. From such initial state, we restarted our simulation and let the fluctuations evolve without energy injection from the reconnection process. Due to the lack of global re- 

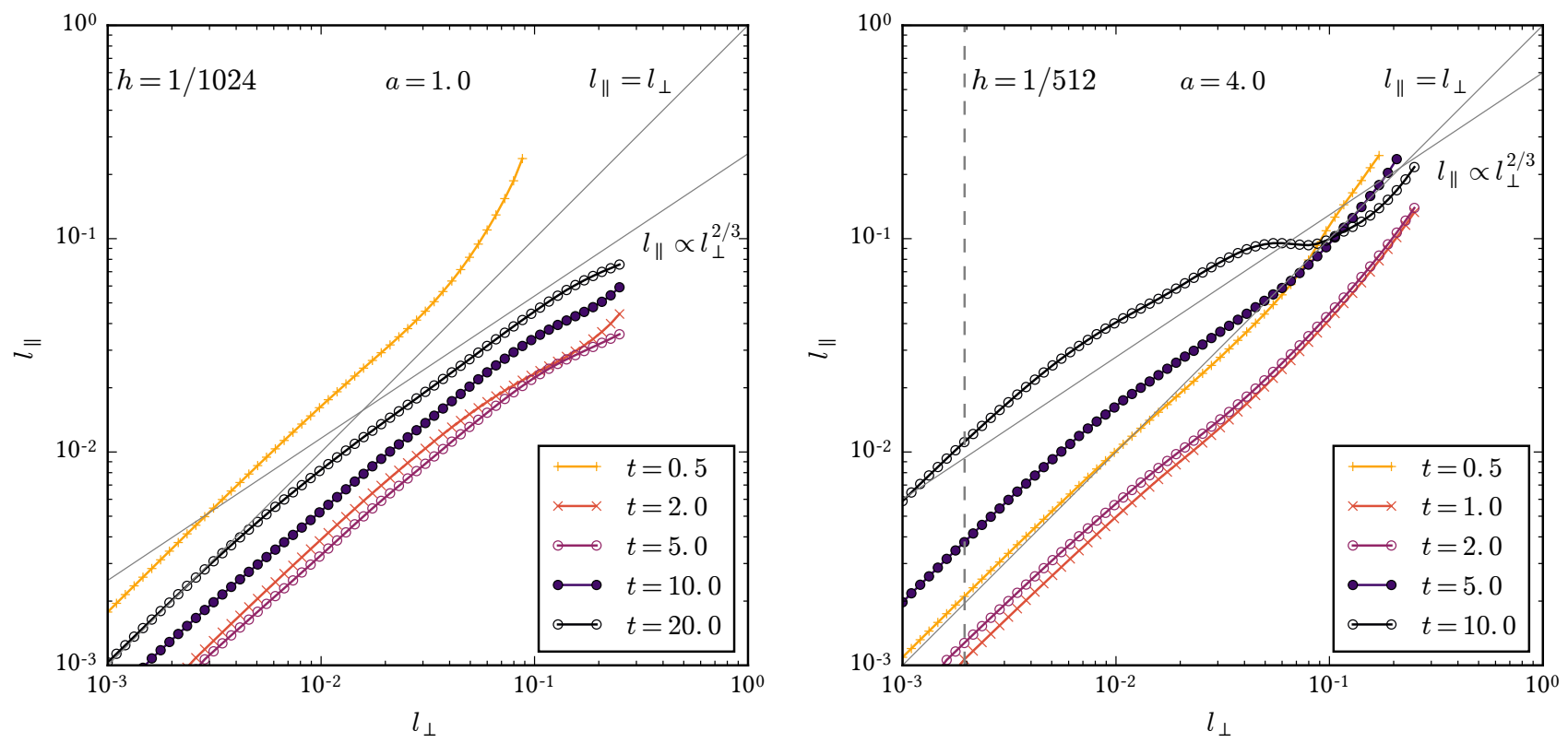

FIG. 7.- Anisotropy scalings for total velocity for models with different sound speeds $(a=1$ on the left and $a=4$ on the right) obtained at different moments. For comparison, we show isotropic and strong turbulence scalings (denoted by gray thin lines). The vertical dashed line in the right plot shows the effective grid size $h=1 / 512$. In the left one, the grid size is below the left axis.

versed field, the reconnection should be strongly suppressed. We should note that the reconnection can still produce weak energy injection from the unchanged components of magnetic field. Nevertheless, the main source of injection should be removed, and previously produced velocity and magnetic field fluctuations should start to decay due to the dissipation. We expect, that due to the lack of reconnection outflows, the fluctuations should quickly produce scaling compatible with the GoldreichSridhar model. We ran this model for a few Alfvén times, and then calculated local structure functions in order to analyze the velocity anisotropy scaling.

In Figure 8 we compare anisotropy scalings for driven and decaying models at the same moment $t=12.0$, i.e. the reconnection-driven turbulence shown with $\times$-points, and the decaying turbulence in which the global magnetic field reversal was removed (open and closed circles for $t=11.0$ and $t=12.0$, respectively). We clearly see the difference in the anisotropy scaling. In the reconnectiondriven case, the outflows still strongly affect the velocity statistics and a scaling closer to $l_{\|} \propto l_{\perp}$ is observed. In the case of decaying turbulence, the reconnection outflows do not affect the statistics anymore and after one Alfvén time the scaling is already aligned with $l_{\|} \propto l_{\perp}^{2 / 3}$ at larger scales. The same is observed at later time $t=12.0$. This test indicates, that the reconnection outflows are responsible for the change of anisotropy scaling.

\section{DISCUSSION}

\subsection{Comparison to previous results}

We performed numerical modeling of reconnectiondriven turbulence and studied its properties. We should note that we did not impose an initial Sweet-Parker or Harris current configurations (as in Lapenta 2008, Oishi et al. 2015; Huang \& Bhattacharjee 2016), but we let a weak noise affect the current sheet along which magnetic field is discontinuous initially. Moreover, in this work we applied periodic boundary conditions along the current sheet, but we left the vertical boundaries open. With the exception of the vertical boundary type, our setup resembles closely the one studied by Beresnyak (2013).

The choice of different vertical boundaries have some important consequences. Periodicity in all three directions with two separated current sheets imposed, as used in Beresnyak (2013), allows for the possibility of horizontal large scale interactions through the deformations of both current sheets. These interactions, especially at later times, can generate large scale motions in the perpendicular direction to the current sheet providing additional to the reconnection energy input(see Drake et al. 2010, Kowal et al. 2011, for a similar setup with many current sheets, in which the current sheet deformations are well manifested). In our case, such large-scale interactions are not allowed, therefore the only energy input comes from the small-scale reconnection events. The reconnection rate values obtained from our models are compatible to those in Beresnyak (2013). We estimated the reconnection rate to be about $0.025-0.035 V_{A}$, while the value estimated in Beresnyak (2013) is around twice smaller. This can be justified by less diffusive spectral code together with smaller resistivity coefficient (of the order of $4 \cdot 10^{-5}$, as estimated from the Lundquist number for their lowest resolution run) used in their work. The spectral slope of the turbulence generated in their models was estimated to be around Kolmogorov slope, which is comparable with the one we show in Figure 5 . Beresnyak (2013) did not demonstrate any results related to the properties of velocity fluctuations, however, and since they focused only on incompressible MHD regime, they did not study $\beta$-dependence.

As we mentioned already, Oishi et al. (2015) used a different numerical setup starting from the Sweet-Parker configuration and periodic box along the current sheet. The Sweet-Parker configuration requires open boundary conditions to allow inflow and outflow of the magnetic 


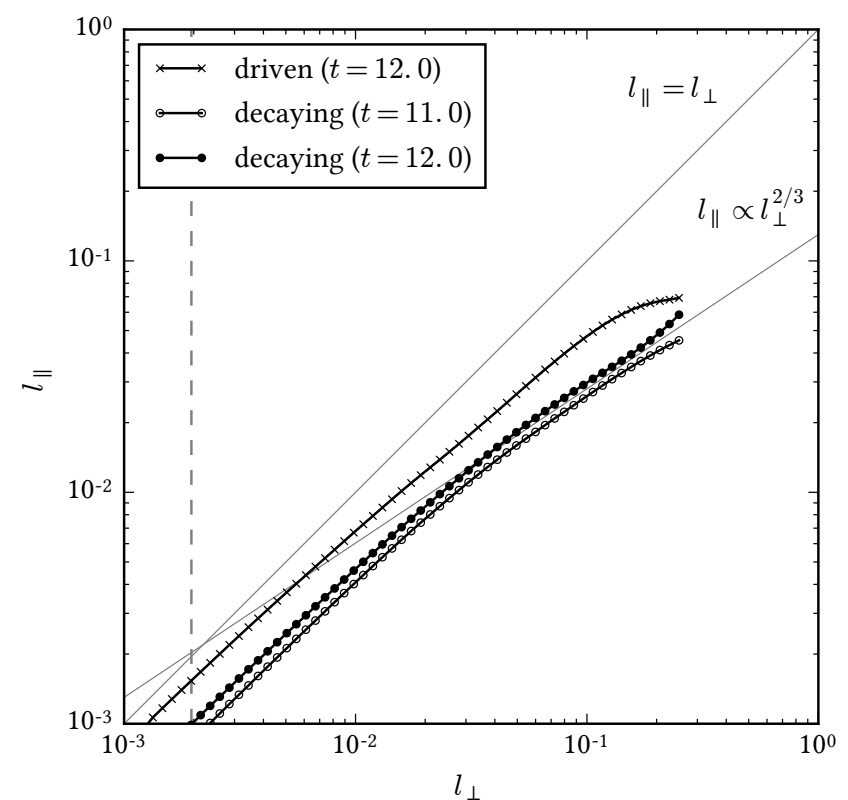

FIG. 8.- Comparison of the anisotropy scalings for velocity in the reconnection-driven turbulence run with the global field reversal at $t=12.0$ ( $\times$ points) and at two time moments $t=11.0$ and 12.0 (open and closed circles, respectively) for model in which the global reversal of magnetic field was removed at $t=10.0$ and the simulation was restarted. We see that while in the reconnectiondriven run the reconnection outflows affect the velocity statistics and the scaling is closer to the isotropic one, in the run without strong outflows (no field reversals) the anisotropy scaling tends to the Goldreich \& Sridhar (1995) scaling, $l_{\|} \propto l_{\perp}^{2 / 3}$, at scales $l_{\perp}>2 \cdot 10^{-2}$.

flux in order to maintain steady state. Since the periodic boundary conditions were applied, the growth of the fluctuations at large scales may be attributed to the presence of the configuration of magnetic field which is not in initial equilibrium. It is also important to notice that these authors have misleadingly claimed to perform very long simulations up to $1000 t_{A}$. They assumed an uncommon definition of Alfvén time $t_{A}=\delta_{0} / V_{A}$, where $\delta_{0}=0.02$ was the current sheet thickness. Typically, the timescale is defined by $t_{A}=L / V_{A}$, where $L$ is the size of the system, which results in their maximum simulation time of $20 t_{A}$, same as in our models. Another important conclusion claimed by Oishi et al. (2015) was that for sufficiently large Lundquist number $S$, the $\delta_{S P}$ becomes weakly dependent on $S$ (see their Figure 1). However, the authors did not provide any estimation of the numerical resistivity $\eta_{\text {num }}$, which we expect to be comparable with the one we estimated, since both studies were performed using Godunov-type codes 9 From Table 1 in Oishi et al. (2015) we can see that for high Lundquist number models, the explicit resistivity $\eta$ is much below $\eta_{\text {num }}$ we estimated. Therefore, the deviation from SweetParker dependence $V_{\text {rec }} \propto S^{-1 / 2}$ observed in their simulation could be artificial and resulting from the numerical effects.

Recently, Huang \& Bhattacharjee (2016) analyzed the statistics of velocity fluctuations generated by reconnection. They also used as a starting point a global Sweet-

9 In Kowal et al. (2009) we estimated that $\eta_{\text {num }} \approx 10^{-4}$ for models with the grid size $h=1 / 512$.

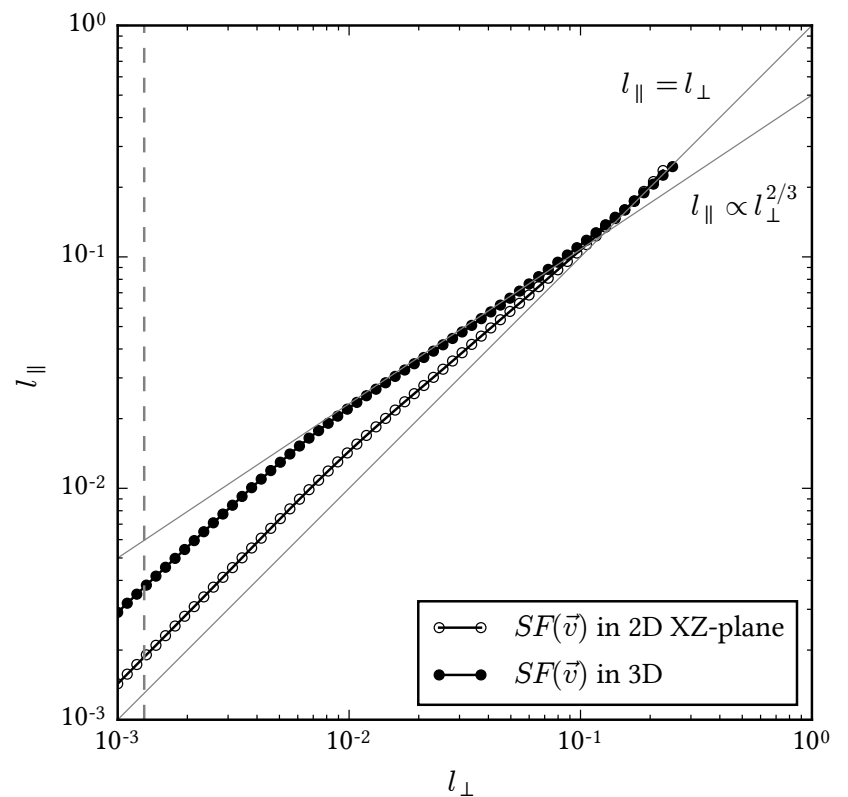

FIG. 9.- Comparison of the anisotropy scalings for velocity in 3D driven turbulence model without the global field reversal obtained from 3D structure function calculated in the local reference frame (solid circles), and the structure functions calculated in 2D XZplanes with the local reference frame defined by the in-plane field (open circles). In the 3D structure function we see perfect scaling compatible with Goldreich \& Sridhar (1995) model from scales $l_{\perp}$ below $10^{-2}$ to above $10^{-1}$. Using the structure function calculated in the XZ-plane with the in-plane defined local reference frame, this scaling is lost. The vertical dashed line corresponds to the grid size $h=1 / 768$. A fully developed turbulent realization at $t=13.0 t_{A}$ was used.

Parker configuration, however, with non-periodic boundaries along the current sheet. Weak velocity perturbations were injected into such domain in order to understand their effect on the reconnection rate. If the perturbations are injected too far from the current sheet, they simply propagate out of the domain as Alfvén waves before reaching the reconnection zone. They used a guide field comparable in strength to the reconnecting component, which additionally prevents the interactions of the waves. They calculated windowed $2 \mathrm{D}$ power spectra of velocity over $X Z$-planes and averaged them over the vertical direction. Their power spectra demonstrated steeper slope comparing to Kolmogorov turbulence. The structure functions were also calculated over $X Z$-planes, and the local reference frame was determined by the in-plane magnetic field. Here, we applied the same technique for calculation of local structure function in turbulent simulations, and found that this method results in much reduced anisotropy, when compared to the fully 3D structure function analysis. In Figure 9 we present the comparison between both methods applied to driven turbulence model without global field reversal simulated in fully periodic box with the grid size $h=1 / 768$. The reduction of the anisotropy scaling due to a different technique is clear. This indicates, that these authors' conclusion of a different type of turbulence (steeper power spectrum and different than GoldreichSridhar anisotropy scaling) should be taken carefully. In our numerical experiments we obtain Kolmogorov-like power spectra of generated velocity fluctuations, which also present Goldreich-Sridhar anisotropy scaling at later 
times in large $\beta$ models. This indicates that the turbulent statistics are similar to strong MHD turbulence, but are strongly affected (contaminated) by the dynamics of reconnection induced flows. In particular this is clearly visible for low $\beta$ models, where supersonic reconnection outflows are present. In high $\beta$ models we observe that the Goldreich-Sridhar anisotropy scaling is visible at much earlier times, since the fluctuations generated by reconnection outflows are propagated faster. We also claim that the Goldreich-Sridhar anisotropy scaling is more apparent for models with higher resolution due to larger dispersion in scales between the turbulent fluctuations and reconnection outflow, what is confirmed by Figure 7. As a consequence, our conclusions differ from those of Huang \& Bhattacharjee (2016) with regards to the type of turbulence in magnetic reconnecting plasmas.

\subsection{Validity of our approach}

In this paper we study magnetic reconnection using magnetohydrodynamic (MHD) description of a plasma (see Section 2). The validity of this description has been broadly discussed in literature (see e.g. Kulsrud 1983 Somov 2006a; Eyink et al. 2011; Lazarian et al. 2015) and its justification can be based on either collisionality or strong magnetization. Following Eyink et al. (2011), where we refer for more detailed review of the validity of MHD description, we consider three characteristic length scales of importance: the ion gyroradius $\rho_{i}$, the ion mean-free-path length $\lambda_{\mathrm{mfp}, i}$ due to Coulomb collisions, and the scale $L$ of large-scale variations of magnetic field and velocity. The fluid picture of a plasma is justified if the plasma is at least somewhat collisional, i.e. $L \gg \lambda_{\mathrm{mfp}, i}$. This regime can be divided into "weakly collisional" $\left(\lambda_{\mathrm{mfp}, i} \gg \rho_{i}\right)$ and "strongly collisional" $\left(\rho_{i} \gg \lambda_{\mathrm{mfp}, i}\right)$ plasmas. In the opposite case, i.e. $\lambda_{\mathrm{mfp}, i} \gg L$, the plasma is "collisionless", and hydrodynamic description does not work anymore. In astrophysical plasmas we can encounter all three regimes of collisionality. For example, plasmas in star interiors and accretion disks are strongly collisional. Very hot and diffuse plasmas, such as warm ionized interstellar medium, are weakly collisional, while solar wind at magnetosphere or post-coronal mass ejection (CME) current sheets are examples of collisionless plasmas (see Table 1 in Eyink et al. 2011). Among the additional assumptions applied to MHD description is the assumption of high conductivity, which is easily fulfilled for most astrophysical plasmas (see e.g. Somov 2006b).

In this work we are interested in reconnection processes at length scales much larger than $\rho_{i}$. For such scales plasmas can be more precisely described by "kinetic MHD equations", which differ from the standard MHD equations by the isotropic thermal pressure $p$ replaced with the pressure tensor, which has two components, $p_{\|}$and $p_{\perp}$, parallel and perpendicular to the local magnetic field, respectively (see e.g. Chew et al. 1956). In the presence of anisotropic pressure, plasma can develop kinetic instabilities, such as "firehose" or "mirror" instabilities, which strongly affect the plasma evolution at small scales (see e.g. Hau \& Wang 2007, and references therein). Our focus in the studies here is to prohibit development of any microscopic instabilities (by using the Particle-inCell or kinetic MHD approaches) or enhanced reconnection rates (by using Hall term or anomalous resistivity), which could influence the generation of turbulence by reconnection process, even if other frameworks describe better the astrophysical plasma of interest in which the reconnection process takes place.

\subsection{Restrictions imposed by periodic boundary conditions}

As we mentioned in Section 2, the choice of periodic boundary conditions along the $\mathrm{X}$ and $\mathrm{Z}$ directions imposes some restrictions on the applicability of the model. For instance, the fluctuations of developed turbulence in the parallel direction to the initial current sheet (the XZplane) cannot reach sizes larger than the computational domain, therefore after around $t \approx t_{A}=V_{A}^{-1}$, the energy could be accumulated at large scales due to the interactions with the domain boundaries and enforce the inertial range production down to the small scales. This, as result, may affect the development of anisotropy. On the other hand, dissipation of the cascade originated waves occurs in similar timescales. One would expect then the energy at small scales to be greatly dominated by the local cascade and less affected by the boundary effects. We performed models with different ratios of the $\mathrm{Z}$ to $\mathrm{X}$ dimensions of the box $\left(L_{z}=0.5 L_{x}\right.$ versus $\left.L_{z}=L_{x}\right)$ and do not see significant changes in the developed spectra and anisotropy statistics. The problem of periodic boundary conditions, however, must be properly addressed and quantified in a follow-up paper.

\subsection{What drives the observed turbulence?}

One of the most important results of this work is the reassurance of self-generation of turbulence in reconnection events. The other is that this turbulence follows standard Kolmogorov and Goldreich-Sridhar statistics. However, a still open issue is related to the driving mechanism of the observed turbulent motions. What drives the turbulence in reconnection events? Literature has suggested, without any quantitative proof, that tearing modes, plasmoid instabilities, and shear-induced instabilities could mediate the energy transfer from coherent to turbulent flows.

In our numerical experiments we do not identify tearing modes, although filamentary plasmoid-like structures are present. The filling factor of these are, however, visually recognized as very small. Sheared flows, on the other hand, are present around and within the whole current sheet. As the field lines reconnect the $\mathbf{v} \times \mathbf{B}+\mathbf{E}$ force increases, accelerating the plasma and creating the current sheet. This process is, in three dimensions, patchy and bursty. Therefore, the accelerated flows are strongly sheared. The statistical importance of these burst flows is large, as already shown in this work, as we compared the velocity anisotropy of reconnecting events to that of decaying turbulence without the reversed field. KelvinHelmholtz instability due to the sheared velocities in reconnecting layers has already been conjectured as possible origin of turbulence by Beresnyak (2013). In this work, we therefore provide real evidence for the selfgenerated turbulence driven by the velocity shear. Nevertheless, a proper analysis of the growth-rates of such instabilities must be conducted in future work.

Another consequence of the mechanism responsible for turbulence self-generation is on the statistics of perturbations. Velocity shear is a global process that occurs in 
regular magnetized and unmagnetized fluids. The nonlinear evolution of related instabilities, as the KelvinHelmholtz instability, is known to be one of the main contributors to the energy transfer rate between wavemodes, i.e. the energy cascade. If the energy cascade in reconnection layers is led by similar mechanisms, it is straightforward to understand why the statistics observed resembles those of Kolmogorov-like turbulence, and GoldreichSridhar anisotropy scaling. In other words, our claim is that the turbulent onset and cascade in reconnection is not different to those found in regular MHD and hydrodynamical systems.

\section{CONCLUSIONS}

We performed numerical modeling of a realistic setup in which magnetic reconnection can develop turbulence from the initial weak noise of velocity fluctuations. We analyzed time evolution of several quantities, including kinetic energy, its distribution among the velocity components, mean vorticity, its filling factor, the thickness of turbulent region, and we estimated the reconnection efficiency using two independent methods. Once the turbulence was developed in a broad region we analyzed its properties using power spectra of velocity and vorticity, and anisotropy scaling of velocity fluctuations with respect to the local mean field.

The conclusions coming from these studies can be summarized in several points:

- Reconnection is able to develop substantial amount of turbulence from initially weak noise of velocity fluctuations. We observe growth of the kinetic energy by over three orders of magnitude.

- The generation of rotational motions measured by vorticity is very quick initially, and reaches saturation after around $5.0 t_{A}$. The saturation level of mean vorticity depends on the grid size $h$ and $\beta$, being higher for models with smaller effective grid size and smaller $\beta$. The filling factor of vorticity with a threshold $|\vec{\omega}| \geq 10.0$ depends also on $h$ and $\beta$, however, for model with the largest $h$, the filling factor decays after reaching a maximum value around $7.0 t_{A}$, while for the highest resolution model it constantly grows until the end of simulation. This indicates that the filling factor of strong rotational motions should be much higher in realistic systems. In models with $L_{z}=L_{x}$ the filling factor of vorticity grows for all models with different sound speeds, even though the effective grid size was $h=1 / 512$.

- The estimated reconnection rates using two inde- pendent methods, i.e. the growth rate of the turbulent region thickness $d \Delta / d t$ and the inflow speed $V_{i n}$ with which the fresh magnetic flux is brought to the system, are independent or weakly dependent on the resolution for similar $\beta$, indicating that the reconnection could be fast without necessity of the presence of external processes, such driven turbulence, or collisionless plasma effects. The dependence of $V_{i n}$ on $\beta$ might be related to additional magnetic field dissipation in supersonic shocks created within the turbulent region.

- The velocity and vorticity present power spectra compatible with Kolmogorov slope. There is a small bump observed at small scales which could be explained by the action of reconnection or sort of bottleneck effect.

- The anisotropy degree and scaling depends on the $\beta$-plasma parameter, related to the timescale of decay of the reconnection outflow interactions to turbulent fluctuations.

- The velocity fluctuations generated by reconnection-driven turbulence are the Goldreich \& Sridhar (1995) model compatible, however, their statistics are strongly distorted by reconnection outflows driving the turbulence, especially for low $\beta$ models, where supersonic motions can be generated. In high $\beta$ models, the velocity anisotropy follows the $l_{\|} \propto l_{\perp}^{2 / 3}$ scaling. This scaling is also visible at large scales for the highest resolution model with $\beta \approx 1.0$.

G.K. acknowledges support from FAPESP (grants no. 2013/04073-2 and 2013/18815-0) and PNPD/CAPES (grant no. 1475088) through a Postdoctoral Fellowship at University Cruzeiro do Sul. This work has made use of the computing facilities of the Laboratory of Astrophysics (EACH/USP, Brazil) and the Academic Supercomputing Center in Kraków, Poland (Supercomputer Prometheus at ACK CYFRONET AGH). D.F.G. thanks the Brazilian agencies CNPq (no. 302949/20143 ) and FAPESP (no. 2013/10559-5) for financial support. A.L. acknowledges the NSF grant AST 1212096, NASA grant NNX14AJ53G as well as a distinguished visitor PVE/CAPES appointment at the Physics Graduate Program of the Federal University of Rio Grande do Norte, the INCT INEspaço and Physics Graduate Program/UFRN. E.T.V. acknowledges the support the AAS.

\section{REFERENCES}

Alfvén, H. 1942, Nature, 150, 405

Armstrong, J. W., Rickett, B. J., \& Spangler, S. R. 1995, ApJ, 443, 209

Beresnyak, A. 2013, ArXiv e-prints, arXiv:1301.7424

Chepurnov, A., Burkhart, B., Lazarian, A., \& Stanimirovic, S. 2015, ApJ, 810, 33

Chepurnov, A., \& Lazarian, A. 2010, ApJ, 710, 853

Chew, G. F., Goldberger, M. L., \& Low, F. E. 1956, Proceedings of the Royal Society of London Series A, 236, 112
Dedner, A., Kemm, F., Kröner, D., et al. 2002, Journal of Computational Physics, 175, 645

Drake, J. F., Opher, M., Swisdak, M., \& Chamoun, J. N. 2010, ApJ, 709, 963

Eyink, G., Vishniac, E., Lalescu, C., et al. 2013, Nature, 497, 466 Eyink, G. L. 2011, Phys. Rev. E, 83, 056405

Eyink, G. L., Lazarian, A., \& Vishniac, E. T. 2011, ApJ, 743, 51

Goldreich, P., \& Sridhar, S. 1995, ApJ, 438, 763

Hau, L.-N., \& Wang, B.-J. 2007, Nonlinear Processes in Geophysics, 14, 557 
Huang, Y.-M., \& Bhattacharjee, A. 2016, ApJ, 818, 20

Jacobson, A. R., \& Moses, R. W. 1984, Phys. Rev. A, 29, 3335

Kirby, J. F. 2005, Computers and Geosciences, 31, 846

Kowal, G. 2016, in prep.

Kowal, G., de Gouveia Dal Pino, E. M., \& Lazarian, A. 2011, ApJ, 735, 102

Kowal, G., Lazarian, A., Vishniac, E. T., \& Otmianowska-Mazur, K. 2009, ApJ, 700, 63

—. 2012, Nonlinear Processes in Geophysics, 19, 297

Kulsrud, R. M. 1983, in Basic Plasma Physics: Selected Chapters, Handbook of Plasma Physics, Volume 1, ed. A. A. Galeev \&

R. N. Sudan, 1

Lapenta, G. 2008, Physical Review Letters, 100, 235001

Lazarian, A. 2005, in American Institute of Physics Conference Series, Vol. 784, Magnetic Fields in the Universe: From Laboratory and Stars to Primordial Structures., ed. E. M. de Gouveia dal Pino, G. Lugones, \& A. Lazarian, 42-53

Lazarian, A., Esquivel, A., \& Crutcher, R. 2012, ApJ, 757, 154

Lazarian, A., Eyink, G., Vishniac, E., \& Kowal, G. 2015,

Philosophical Transactions of the Royal Society of London Series A, 373, 20140144

Lazarian, A., Kowal, G., Takamoto, M., de Gouveia Dal Pino, E. M., \& Cho, J. 2016, in Astrophysics and Space Science Library, Vol. 427, Astrophysics and Space Science Library, ed. W. Gonzalez \& E. Parker, 409

Lazarian, A., \& Vishniac, E. T. 1999, ApJ, 517, 700

Lazarian, A., \& Vishniac, E. T. 2009, in Revista Mexicana de Astronomia y Astrofisica Conference Series, Vol. 36, Revista Mexicana de Astronomia y Astrofisica Conference Series, 81-88
Matthaeus, W. H., \& Lamkin, S. L. 1985, Physics of Fluids, 28, 303

-. 1986, Physics of Fluids, 29, 2513

Mignone, A. 2007, Journal of Computational Physics, 225, 1427

Oishi, J. S., Mac Low, M.-M., Collins, D. C., \& Tamura, M. 2015, ApJ, 806, L12

Padoan, P., Juvela, M., Kritsuk, A., \& Norman, M. L. 2009, ApJ, 707, L153

Parker, E. N. 1957, J. Geophys. Res., 62, 509

Petschek, H. E. 1964, NASA Special Publication, 50, 425

Priest, E., \& Forbes, T. 2007, Magnetic Reconnection

Ruuth, S. J. 2006, Mathematics of Computation, 75, 183

Santos-Lima, R., Lazarian, A., de Gouveia Dal Pino, E. M., \&

Cho, J. 2010, ApJ, 714, 442

Somov, B. V., ed. 2006a, Astrophysics and Space Science Library, Vol. 340, Plasma astrophysics, part I : fundamentals and practice

—. 2006b, Astrophysics and Space Science Library, Vol. 341,

Plasma astrophysics, part II : reconnection and flares

Sweet, P. A. 1958, The Observatory, 78, 30

Takamoto, M., Inoue, T., \& Lazarian, A. 2015, ApJ, 815, 16

Vishniac, E. T., \& Lazarian, A. 1999, ApJ, 511, 193 